\title{
Isolated rhombencephalitis with good clinical recovery
}

\section{Rombencefalite isolada com boa recuperação clínica}

Bruna Klein da Costaำ, Bruno Samuel Fraiman de Oliveira ${ }^{1}$, Jefferson Becker ${ }^{1}$, Douglas Kazutoshi Sato ${ }^{123}$

A 20-year-old, previously-healthy woman presented with progressive tetraparesis, multiple cranial nerve involvement and pseudobulbar affect over three weeks. Brain MRI revealed a unique edematous brainstem lesion with peripheral gadolinium enhancement (Figure 1). She was treated with long-term antibiotics (21 days of ampicillin and sulfamethoxazole/trimethoprim) and high-dose intravenous corticosteroids. Oral prednisone was slowly tapered with full clinical recovery and resolution of the lesion after five months (Figure 2).

Idiopathic inflammatory central nervous system disease and neuroinfection are the major differential diagnoses for isolated rhombencephalitis, especially Listeria ${ }^{1}$. Despite the severity, patients may have full recovery if managed properly.
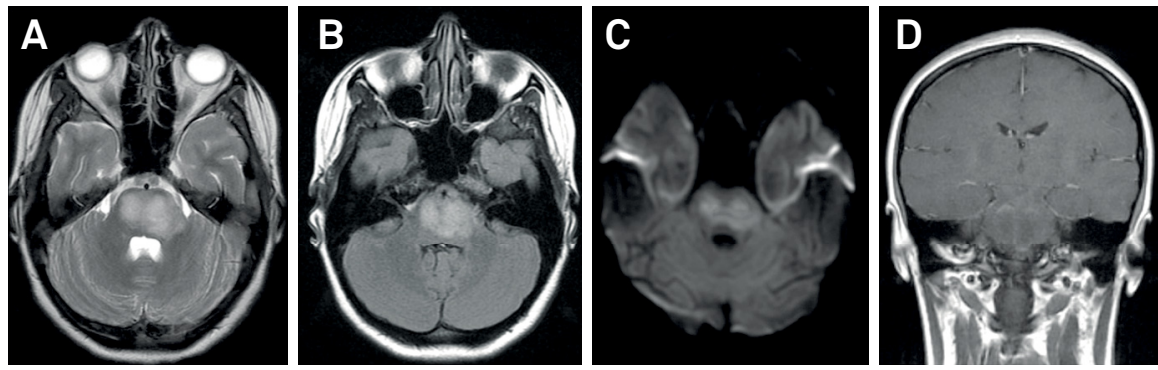

Figure 1. Brain MRI. (A) Axial T2 weighted and fluid-attenuated inversion recovery (FLAIR) (B) images demonstrating a hyperintense brainstem lesion with increased signal in diffusion-weighted image (DWI) (C). Coronal T1 weighted post-gadolinium image (D) show mild peripheral enhancement.
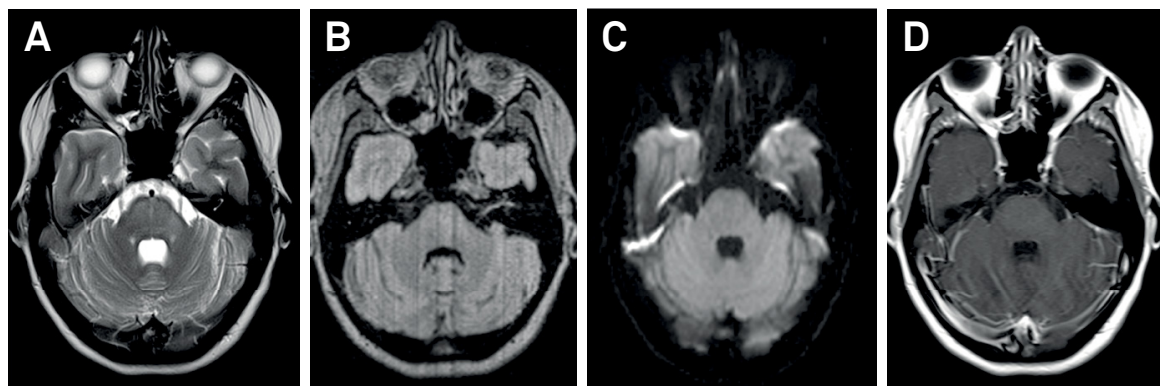

Figure 2. Brain MRI after treatment. Axial T2 weighted (A), FLAIR (B) and DWI (C) sequences demonstrate almost complete resolution of the brainstem lesion. Axial T2 weighted image shows discrete hyperintense lesion (A) without gadolinium enhancement (D).

\section{References}

1. Jubelt B, Mihai C, LiTM, Veerapaneni P. Rhombencephalitis /

brainstem encephalitis. Curr Neurol Neurosci Rep. 2011;11(6):543-52.

https://doi.org/10.1007/s11910-011-0228-5

\footnotetext{
'Pontifícia Universidade Católica do Rio Grande do Sul, Hospital São Lucas, Serviço de Neurologia, Porto Alegre RS, Brasil; 2Universidade de São Paulo, Faculdade de Medicina, Departamento de Neurologia, São Paulo SP, Brasil;

${ }^{3}$ Tohoku University Graduate School of Medicine, Department of Multiple Sclerosis Therapeutics, Sendai, Japan.

Correspondence: Douglas Kazutoshi Sato; Serviço de Neurologia do Hospital São Lucas / PUCRS; Av. Ipiranga, 6690; 90610-000 Porto Alegre RS, Brasil;

E-mail:douglas.sato@med.tohoku.ac.jp

Conflict of interest: There is no conflict of interest to declare.

Received 18 December 2016; Accepted 18 July 2017.
} 